\title{
The Sphere Motion via SLERP
}

\section{Hatice KUŞAK SAMANCI ${ }^{1}$}

${ }^{I}$ Bitlis Eren University, Faculty of Arts and Sciences, Department of Mathematics, 13000 Bitlis / TURKEY

\section{Abstract}

In this paper, we investigate a brief survey on the three-sphere motion by using the quaternion interpolasyon SLERP. Firstly, we consider the moving and fixed quaternion frames for three-sphere motion onto a unit quaternionic sphere. Then we calculate the equations of the velocity and we investigate some properties of the canonical relative system. Finaly we give some examples for these equations.

\section{Key Words}

Quaternion, Spherical Motion, SLERP 


\section{INTRODUCTION}

Quaternions are found by Sir William Rowan Hamilton in the midnineteenth century to generalize complex numbers in some way that would be applicable to three-dimensional 3D space, (Hamilton,1853). The quaternion has a scalar component and three imaginary components. If a quaternion given by $q=\left(q_{0}, \mathbf{q}\right)$ obeys the constraint $q \cdot q=1$, the locus of these points is the hypersphere $\mathrm{S}^{3}$. The most important property of quaternions is that every unit quaternion represents a rotation in three dimensional space. Since the rotation matrix is a very complex structure, the simple structure of the quaternions ensures that we can make the three-dimensional rotation movement easier.

The spherical motion was previously studied by Garnier in (Garnier,1956) and obtained spherical Euler-Savary equation by H. Müller in (Müller,1963). He calculated the motion velocities and pole curves of these motions in Euclidean space. Thus, the quaternions have been firstly investigated for the spherical kinematic by Blaschke in 1960, (Blaschke,1960). Some basic preliminaries of theorical kinematics, three-spheres and quaternions can be found in [(Hacısalihoğlu,1983), (Hacısalihoğlu,1983), (Hanson,2006)]. In the mechanical and robotic engineering, spherical motion is useful for every motion of the spherical mechanism. We can see some papers related with applications of spherical motion for spherical mechanisms in [(Larochelle,2000), (Liu et al., 2003), (Yang et al., 1964), (Alizade et al., 2005), (Kuşak et al., 2011),(Shoemake, 1985)]. Although the quaternions have been used in some scientific areas as physics, kinematics, mechanics, robotics and etc., nowadays the quaternions have been began to use commonly in the computer graphics and computer game animation. The methods of quaternion interpolations were defined to the graphics community originally by Shomake in the papers [(Shoemake, 1985), (Shoemake, 1987)]. By analogy to the acronym "LERP" that might be used for ordinary linear interpolation, Shomake coined the term "SLERP" for "spherical linear interpolation", a terminology that remains in common usage. The SLERP can be transformed to provide close analogs of the anchor-point and tangent-direction properties of the conventional families of Euclidean splines. It is reasonably straightforward to develop uniform quaternion spline families in an elegant practical form, and he discussed quaternion splines thoroughly. The fundamental concepts of quaternions, methods of quaternion visualization, applications of SLERP etc., in (Hanson, 2006). We have seen that the motion of the quaternion spheres is not examined before with SLERP. In our study, quaternion sphere motion was investigated with SLERPler for the first time. In the preliminary section, some basic concepts about the three-sphere, the quaternion algebra, the SLERP and the matrix representation of the rotation are given. In the main results, the orthonormal quaternion frames $\left\{\mathrm{O}, \mathrm{e}_{0}, \mathrm{e}_{1}, \mathrm{e}_{2}, \mathrm{e}_{3}\right\}$ and $\left\{\mathrm{O}, \mathrm{e}_{0}^{\prime}, \mathrm{e}_{1}^{\prime}, \mathrm{e}_{2}{ }_{2}, \mathrm{e}_{3}^{\prime}\right\}$ are taken to represent moving three-sphere $\mathrm{S}$ and fixed three sphere $S^{\prime}$ in the four dimensional space, respectively. Then another orthonormal quaternion frame $\left\{O, q_{0}, q_{1}, q_{2}, q_{3}\right\}$ is taken, and this quaternion frame is called relative orthonormal quaternion frame. Furthermore, we calculate the equations of the motion velocities of one parameter three-spherical motions. Also, some relations about pole curves are obtained by using SLERP. At the end of the study, we give some examples about the concept.

\section{PRELIMINARIES}

\subsection{Three-sphere with The Quaternion Notation}

Three-sphere which consists of the set of points equidistant from a fixed central point in four dimensional Euclidean space is a higher-dimensional of a sphere. In coordinates, a three-sphere with center $\left(C_{0}, C_{1}, C_{2}, C_{3}\right)$ and radius $\mathrm{r}$ is the set of all points $\left(x_{0}, x_{1}, x_{2}, x_{3}\right)$ in real, four dimensional space $\mathbb{R}^{4}$ such that

$\sum_{i=0}^{3}\left(x_{i}-C_{i}\right)^{2}=r^{2}$

The three-sphere centered at the origin with $r=1$

$$
S^{3}=\left\{\left(x_{0}, x_{1}, x_{2}, x_{3}\right) \in \mathbb{R}^{4}: x_{0}^{2}+x_{1}^{2}+x_{2}^{2}+x_{3}^{2}=1\right\}
$$

is defined the unit three sphere. The three-sphere is also represented by quaternion set $\mathbb{H}$. Therefore the unit three-sphere is given by using the quaternions as $S^{3}=\{q \in \mathbb{H}:\|q\|=1\}$, (Hanson,2006).

\subsection{Quaternions Algebra}

Consider the quaternion variable $q=q_{0} \cdot e_{0}+q_{1} \cdot e_{1}+q_{2} \cdot e_{2}+q_{3} \cdot e_{3} \in \mathbb{H}$, where $q_{0}, q_{1}, q_{2}$ and $q_{3}$ are real-valued scalars, and here $e_{0}, e_{1}, e_{2}, e_{3}$ are orthogonal unit vectors and imaginary units, such that $e_{0}^{2}=1, e_{1}^{2}=e_{2}^{2}=e_{3}^{2}=-1, e_{1} e_{2}=-e_{2} e_{1}=e_{3}$, $e_{3} e_{2}=-e_{2} e_{3}=e_{1}, e_{3} e_{1}=-e_{1} e_{3}=e_{2}$. It is clear that $\mathbb{H}$ is an associative, but not commutative algebra. The quaternion can also be denoted in terms of its scalar part $S_{q}$ and vector part $\mathbf{V}_{q}$, such that $q=S_{q}+\mathbf{V}_{q}$. If $q=q_{0} \cdot e_{0}+q_{1} \cdot e_{1}+q_{2} \cdot e_{2}+q_{3} \cdot e_{3}$ and 
$p=p_{0} \cdot e_{0}+p_{1} \cdot e_{1}+p_{2} \cdot e_{2}+p_{3} \cdot e_{3}$ are quaternions, the addition, subtraction and multiplication of the quaternions are defined by

$$
\begin{aligned}
& q+p=\left(S_{q}+S_{p}\right)+\left(\mathbf{V}_{q}+\mathbf{V}_{p}\right) \\
& q \cdot p=S_{q} S_{p}-<\mathbf{V}_{q}, \mathbf{V}_{p}>+S_{q} \mathbf{V}_{p}+S_{p} \mathbf{V}_{q}+\mathbf{V}_{q} \times \mathbf{V}_{p} .
\end{aligned}
$$

Also, the multiplication of quaternions in Eq (1) can be written in matrix form as

$$
p . q=\left[\begin{array}{c}
p_{0} q_{0}-p_{1} q_{1}-p_{2} q_{2}-p_{3} q_{3} \\
p_{1} q_{0}+p_{0} q_{1}+p_{2} q_{3}-p_{3} q_{2} \\
p_{2} q_{0}+p_{0} q_{2}+p_{3} q_{1}-p_{1} q_{3} \\
p_{3} q_{0}+p_{0} q_{3}+p_{1} q_{2}-p_{2} q_{1}
\end{array}\right]=\left[\begin{array}{cccc}
p_{0} & -p_{1} & -p_{2} & -p_{3} \\
p_{1} & p_{0} & -p_{3} & p_{2} \\
p_{2} & p_{3} & p_{0} & -p_{1} \\
p_{3} & -p_{2} & p_{1} & p_{0}
\end{array}\right]\left[\begin{array}{c}
q_{0} \\
q_{1} \\
q_{2} \\
q_{3}
\end{array}\right] .
$$

Here, specially if $S_{q}=0$ and $S_{p}=0$, the quaternion is called "pure quaternion". The multiplication of pure quaternions are calculated by

$$
q \cdot p=-<\mathbf{V}_{q}, \mathbf{V}_{p}>+\mathbf{V}_{q} \times \mathbf{V}_{p} .
$$

The conjugate $\bar{q}$ of the quaternion $q$ is $q=q_{0} \cdot e_{0}-q_{1} \cdot e_{1}-q_{2} \cdot e_{2}-q_{3} \cdot e_{3}=S_{q}-\mathbf{V}_{q}$. The norm of the quaternion $q$ is defined by $N_{q}=\sqrt{q \cdot \bar{q}}=\sqrt{q_{0}^{2}+q_{1}^{2}+q_{2}^{2}+q_{3}^{2}}$. If the norm is unit, i.e. $N_{q}=1$, then the quaternion is defined "unit quaternion". The unit quaternion $q$ is denoted by the formula $q=\left(\cos \frac{\theta}{2}, \hat{\mathbf{n}} \sin \frac{\theta}{2}\right)$, too. This notation shows that a unit quaternion corresponds to the standart rotation matrix

$\mathbf{R}(\theta, \hat{\mathbf{n}})=\left[\begin{array}{ccc}\cos \theta+n_{1}^{2}(1-\cos \theta) & n_{1} n_{2}(1-\cos \theta)-n_{3} \sin \theta & n_{1} n_{3}(1-\cos \theta)+n_{2} \sin \theta \\ n_{2} n_{1}(1-\cos \theta)+n_{3} \sin \theta & \cos \theta+n_{2}^{2}(1-\cos \theta) & n_{2} n_{3}(1-\cos \theta)-n_{1} \sin \theta \\ n_{3} n_{1}(1-\cos \theta)-n_{2} \sin \theta & n_{3} n_{2}(1-\cos \theta)+n_{1} \sin \theta & \cos \theta+n_{3}^{2}(1-\cos \theta)\end{array}\right]$.

by an angle $\theta$ about the direction $\hat{\mathbf{n}}=\left(n_{1}, n_{2}, n_{3}\right)$. Furthermore, we can write the above rotation matrix with the quaternion components as

$$
\mathbf{R}(q)=\left[\begin{array}{ccc}
q_{0}^{2}+q_{1}^{2}-q_{2}^{2}-q_{3}^{2} & 2 q_{1} q_{2}-2 q_{0} q_{3} & 2 q_{1} q_{3}+2 q_{0} q_{2} \\
2 q_{1} q_{2}+2 q_{0} q_{3} & q_{0}^{2}-q_{1}^{2}+q_{2}^{2}-q_{3}^{2} & 2 q_{2} q_{3}-2 q_{0} q_{1} \\
2 q_{1} q_{3}-2 q_{0} q_{1} & 2 q_{2} q_{3}+2 q_{0} q_{1} & q_{0}^{2}-q_{1}^{2}-q_{2}^{2}+q_{3}^{2}
\end{array}\right] .
$$

where $q_{0}, q_{1}, q_{2}, q_{3} \in \mathbb{R}$, [(Hamilton, 1853), (Blaschke,1960), (Hacisalihoğlu,1983), (Hanson,2006)]. One of the interpolation methods with using the quaternion is spherical linear interpolation (SLERP) defined by

$$
q(t)=\operatorname{SLERP}\left(q_{A}, q_{B}, t\right)=q_{A} \frac{\sin ((1-t) \phi)}{\sin \phi}+q_{B} \frac{\sin (t \phi)}{\sin \phi},
$$

where $q_{A}$ and $q_{B}$ are two quaternionic points on three-sphere.

If specially the starting and ending quaternions are taken as $q_{A}=(1,0,0,0)$ and $q_{B}=\left(\cos \frac{\theta}{2}, \mathbf{n} \sin \frac{\theta}{2}\right)$, then the interpolation is defined by

$$
q(t)=\operatorname{SLERP}\left(q_{A}, q_{B}, t\right)=\left(\cos \left(\frac{t \theta}{2}\right), \mathbf{n} \sin \left(\frac{t \theta}{2}\right)\right),
$$

where $\cos \phi=\cos \frac{\theta}{2}$, (Hanson,2006). 


\section{MAIN RESULTS}

\subsection{The Three-spherical Motions By Quaternions}

Let us think of the spherical movement that the concentric moving $K$ sphere and the constant $K^{\prime}$ sphere has formulated according to each other. Quaternion frame representations belonging to the moving $K$ sphere and the constant $K^{\prime}$ sphere, both owning a given $\mathrm{O}$ center point, would be $\left\{O, e_{0}, e_{1}, e_{2}, e_{3}\right\}$ and $\left\{O, e_{0}{ }_{0}, e_{1}{ }_{1}, e_{2}{ }_{2}, e^{\prime}{ }_{3}\right\}$ respectively. Properties of the quaternion bases for the $\left\{O, e_{0}, e_{1}, e_{2}, e_{3}\right\}$ system are $e_{0}^{2}=1, e_{1}^{2}=e_{2}^{2}=e_{3}^{2}=-1 ; e_{1} e_{2}=e_{3}, e_{3} e_{2}=e_{1}, e_{3} e_{1}=e_{2}$. Properties of the quaternion bases for the $\left\{O, e_{0}^{\prime}, e_{1}^{\prime}, e_{2}^{\prime}, e_{3}^{\prime}\right\}$ system are $\left(e_{0}^{\prime}\right)^{2}=1,\left(e_{1}^{\prime}\right)^{2}=\left(e_{2}^{\prime}\right)^{2}=\left(e_{3}^{\prime}\right)^{2}=-1$ and $e_{1}^{\prime} e_{2}^{\prime}=e_{3}^{\prime}, e_{3}^{\prime} e_{2}^{\prime}=e_{1}^{\prime}$, $e_{3}^{\prime} e_{1}^{\prime}=e_{2}^{\prime}$. Alternatively, we can utilize the quaternion multiplications to form the basis vectors. We can obtain the basis quaternions by rotating the $x, y, z$ axes of coordinate via $\tilde{q}=\left(\tilde{q}_{0}, \tilde{q}_{1}, \tilde{q}_{2}, \tilde{q}_{3}\right)=\cos \frac{\tilde{\theta}}{2}+\tilde{\mathbf{n}} \sin \frac{\tilde{\theta}}{2} \quad$ and $\hat{q}=\left(\hat{q}_{0}, \hat{q}_{1}, \hat{q}_{2}, \hat{q}_{3}\right)=\cos \frac{\hat{\theta}}{2}+\hat{\mathbf{n}} \sin \frac{\hat{\theta}}{2}$ quaternions. In the present case, the quaternion frames of the moving $K$ sphere and the constant $K^{\prime}$ sphere would be as;

$$
\begin{aligned}
& e_{0}=\tilde{q}(1, \mathbf{0}) \tilde{q}^{-1} \quad e^{\prime}{ }_{0}=\hat{q}(1, \mathbf{0}) \hat{q}^{-1} \\
& e_{1}=\tilde{q}(0, \mathbf{x}) \tilde{q}^{-1} \quad e^{\prime}{ }_{1}=\hat{q}(0, \mathbf{x}) \hat{q}^{-1} \\
& e_{2}=\tilde{q}(0, \mathbf{y}) \tilde{q}^{-1} \quad \text { and } \quad e^{\prime}{ }_{2}=\hat{q}(0, \mathbf{y}) \hat{q}^{-1} \\
& e_{3}=\tilde{q}(0, \mathbf{z}) \tilde{q}^{-1} \quad e^{\prime}{ }_{3}=\hat{q}(0, \mathbf{z}) \hat{q}^{-1} \text {. }
\end{aligned}
$$

Consecutively, the matrix that is formed by the $\left[e_{0} e_{1} e_{2} e_{3}\right]$ quaternionic bases belonging to the moving $K$ sphere would be as;

$$
\left[\begin{array}{cccc}
\tilde{q}_{0}^{2}+\tilde{q}_{1}^{2}+\tilde{q}_{2}^{2}+\tilde{q}_{3}^{2} & 0 & 0 & 0 \\
0 & \tilde{q}_{0}^{2}+\tilde{q}_{1}^{2}-\tilde{q}_{2}^{2}-\tilde{q}_{3}^{2} & 2 \tilde{q}_{1} \tilde{q}_{2}-2 \tilde{q}_{0} \tilde{q}_{3} & 2 \tilde{q}_{1} \tilde{q}_{3}+2 \tilde{q}_{0} \tilde{q}_{2} \\
0 & 2 \tilde{q}_{1} \tilde{q}_{2}+2 \tilde{q}_{0} \tilde{q}_{3} & \tilde{q}_{0}^{2}-\tilde{q}_{1}^{2}+\tilde{q}_{2}^{2}-\tilde{q}_{3}^{2} & 2 \tilde{q}_{2} \tilde{q}_{3}-2 \tilde{q}_{0} \tilde{q}_{1} \\
0 & 2 \tilde{q}_{1} \tilde{q}_{3}-2 \tilde{q}_{0} \tilde{q}_{1} & 2 \tilde{q}_{2} \tilde{q}_{3}+2 \tilde{q}_{0} \tilde{q}_{1} & \tilde{q}_{0}^{2}-\tilde{q}_{1}^{2}-\tilde{q}_{2}^{2}+\tilde{q}_{3}^{2}
\end{array}\right] .
$$

The matrix that is formed by the $\left[e_{0}^{\prime} e_{1}^{\prime} e_{2}^{\prime} e_{3}^{\prime}\right]$ quaternionic bases belonging to the constant $K^{\prime}$ sphere would be calculated as;

$$
\left[\begin{array}{cccc}
\hat{q}_{0}^{2}+\hat{q}_{1}^{2}+\hat{q}_{2}^{2}+\hat{q}_{3}^{2} & 0 & 0 & 0 \\
0 & \hat{q}_{0}^{2}+\hat{q}_{1}^{2}-\hat{q}_{2}^{2}-\hat{q}_{3}^{2} & 2 \hat{q}_{1} \hat{q}_{2}-2 \hat{q}_{0} \hat{q}_{3} & 2 \hat{q}_{1} \hat{q}_{3}+2 \hat{q}_{0} \hat{q}_{2} \\
0 & 2 \hat{q}_{1} \hat{q}_{2}+2 \hat{q}_{0} \hat{q}_{3} & \hat{q}_{0}^{2}-\hat{q}_{1}^{2}+\hat{q}_{2}^{2}-\hat{q}_{3}^{2} & 2 \hat{q}_{2} \hat{q}_{3}-2 \hat{q}_{0} \hat{q}_{1} \\
0 & 2 \hat{q}_{1} \hat{q}_{3}-2 \hat{q}_{0} \hat{q}_{1} & 2 \hat{q}_{2} \hat{q}_{3}+2 \hat{q}_{0} \hat{q}_{1} & \hat{q}_{0}^{2}-\hat{q}_{1}^{2}-\hat{q}_{2}^{2}+\hat{q}_{3}^{2}
\end{array}\right] .
$$

Now let us approach the third $\left\{O, q_{0}, q_{1}, q_{2}, q_{3}\right\}$ quaternionic frame to examine the spherical motion. Here, we would get the conditions $q_{0}=1, \quad q_{1}^{2}=q_{2}^{2}=q_{3}^{2}=-1, q_{1} q_{2}=q_{3}, q_{3} q_{2}=q_{1}, q_{3} q_{1}=q_{2}$ from the quaternion properties. We can also form this quaternion via;

$$
\begin{array}{ll}
q_{0}=\widehat{q}(1, \mathbf{0}) \hat{q}^{-1}, & q_{1}=\widehat{q}(0, \mathbf{x}) \hat{q}^{-1} \\
q_{2}=\widehat{q}(0, \mathbf{y}) \hat{q}^{-1}, & q_{3}=\widehat{q}(0, \mathbf{z}) \hat{q}^{-1} .
\end{array}
$$

Matrix of the third quaternion frame $\left[q_{0} q_{1} q_{2} q_{3}\right]$ would be represented as;

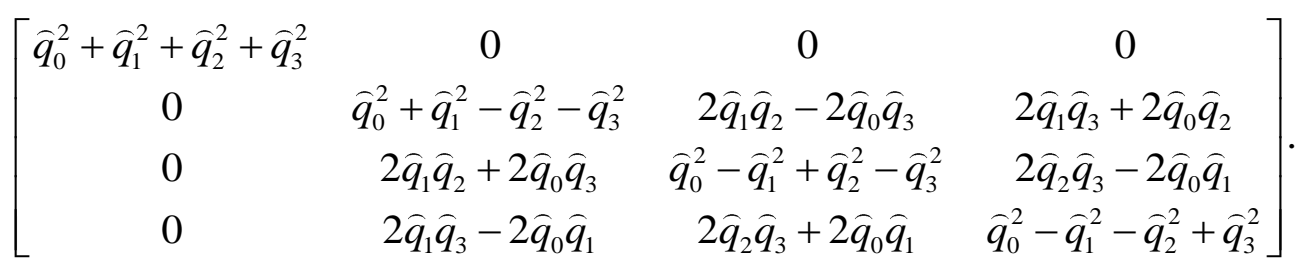

Each three quaternion frame system would be in the same rotation direction, 


$$
\begin{aligned}
& (1, \mathbf{0})=\tilde{q}^{-1} e_{0} \tilde{q}=\hat{q}^{-1} e_{0} \hat{q}=\hat{q}^{-1} e_{0} \hat{q}, \quad(0, \mathbf{x})=\tilde{q}^{-1} e_{1} \tilde{q}=\hat{q}^{-1} e_{1} \hat{q}=\hat{q}^{-1} e_{1} \hat{q} \\
& (0, \mathbf{y})=\tilde{q}^{-1} e_{2} \tilde{q}=\hat{q}^{-1} e_{2} \hat{q}=\hat{q}^{-1} e_{2} \hat{q}, \quad(0, \mathbf{z})=\tilde{q}^{-1} e_{3} \tilde{q}=\hat{q}^{-1} e_{3} \hat{q}=\hat{q}^{-1} e_{3} \hat{q} .
\end{aligned}
$$

As seen from the above equations, it is possible to transfer from one system to another using the rotations around the $\mathrm{O}$ point. Thus, we would obtain the following linear transformations:

$q_{j}=\sum_{k=0}^{3} a_{j k} e_{k}, \quad q_{j}=\sum_{k=0}^{3} a_{j k}^{\prime} e_{k}^{\prime}, \quad j=0,1,2,3$

Here, the $\left\|a_{j k}\right\|,\left\|a^{\prime}{ }_{j k}\right\|$ matrices are idiosyncratic and orthogonal, they respectively represent a transfer from the frames of axis that were established in $K$ and $K^{\prime}$ to the attribution system. If the coefficients $a_{j k}, a^{\prime}{ }_{j k}$ are the proper functions of a $t$ real parameter, we would obtain a "one-parameter spherical motion" of $K$ as opposed to $K$ '. Here, we realize that the coefficients $a_{j k}, a^{\prime}{ }_{j k}$ are not only constant, but they are also differentiable to any desired order. In this regard, the described motion is named briefly as the "one-parameter $D_{I}$ rotational motion" around O.

The changes of the $q_{j}$ quaternions in the $K$ and $K^{\prime}$ spherical motions are respectively represented in the following equations;

$d q_{j}=\sum_{k=0}^{3} e_{k} d a_{j k} \quad$ and $\quad d^{\prime} q_{j}=\sum_{k=0}^{3} e^{\prime}{ }_{k} d a^{\prime}{ }_{j k}$

Here the representations $d a_{j k}, d a^{\prime}{ }_{j k}$ are full differentials based on one or two variables, and the quaternions $e_{k}$ and $e_{k}^{\prime}$ are constant. In this case, the differentials $d q_{j}$ and $d^{\prime} q_{j}$ can again be represented via quaternions $q_{0}, q_{1}, q_{2}, q_{3}$. To do this, equation (6) must be solved according to $e_{k}$ and $e_{k}^{\prime}$, the obtained values must then be properly put into equation (7). On the other hand, $\omega=|\boldsymbol{\omega}|$ is the angular velocity where $\boldsymbol{\omega}=\left(\omega_{1}, \omega_{2}, \omega_{3}\right)$ is the direction of angular velocity. The polar representation of the quaternion is $q(t)=\left(\cos \left(\frac{\omega t}{2}\right), \omega \sin \left(\frac{\omega t}{2}\right)\right)$. Thus the differential equation of the polar representation can be shown by the following equation;

$d q(t)=\frac{1}{2} q(0, \omega)$.

The following equations by using the differential of equation $q q^{-1}=(1,0)$ are obtained

$d q=q\left(q^{-1} d q\right)=\frac{1}{2} q(0, \boldsymbol{\omega})$

$d q^{-1}=\left(d q^{-1} q\right) q^{-1}=-\left(q^{-1} d q\right) q^{-1}=-\frac{1}{2}(0, \boldsymbol{\omega}) q^{-1}$,

Here, we can see that the calculation will be made using the equations given above;

$\boldsymbol{\omega}=2\left(q_{0} d q-q d q_{0}-q \times d q\right)$,

see in (Hanson,2006). If we are to use the differential equation (5) of the quaternion mentioned above to get the differential of the column elements of the matrix, we would obtain the following;

$$
\begin{aligned}
d e_{1} & =d q(0, \mathbf{x}) q^{-1}+q(0, \mathbf{x}) d q^{-1} \\
& =\left[\frac{1}{2} q(0, \boldsymbol{\omega})\right](0, \mathbf{x}) q^{-1}+q(0, \mathbf{x})\left[-\frac{1}{2}(0, \boldsymbol{\omega}) q^{-1}\right] \\
& =\frac{1}{2} q[(0, \boldsymbol{\omega})(0, \mathbf{x})-(0, \mathbf{x})(0, \boldsymbol{\omega})] q^{-1} \\
& =q(0, \boldsymbol{\omega} \times \mathbf{x}) q^{-1} .
\end{aligned}
$$

Similarly, calculations for other bases give the following differentials; 


$$
\begin{aligned}
& d e_{0}=q(0, \boldsymbol{\omega} \times \mathbf{0}) q^{-1}, d e_{1}=q(0, \boldsymbol{\omega} \times \mathbf{x}) q^{-1} \\
& d e_{2}=q(0, \boldsymbol{\omega} \times \mathbf{y}) q^{-1}, d e_{3}=q(0, \boldsymbol{\omega} \times \mathbf{z}) q^{-1} .
\end{aligned}
$$

The differentiation matrix of the bases $q_{i},(i=0,1,2,3)$ of the $q$ unit quaternion in the $K$ sphere, and the corresponding matrix is calculated by the following;

$$
\left[\begin{array}{l}
d q_{0} \\
d q_{1} \\
d q_{2} \\
d q_{3}
\end{array}\right]=\frac{1}{2}\left[\begin{array}{cccc}
0 & -\omega_{1} & -\omega_{2} & -\omega_{3} \\
\omega_{1} & 0 & \omega_{3} & -\omega_{2} \\
\omega_{2} & -\omega_{3} & 0 & \omega_{1} \\
\omega_{3} & \omega_{2} & -\omega_{1} & 0
\end{array}\right]\left[\begin{array}{l}
q_{0} \\
q_{1} \\
q_{2} \\
q_{3}
\end{array}\right] .
$$

By a similar method, the following differential equation is used to obtain the changes of the bases in the $K^{\prime}$ sphere;

$$
\left[\begin{array}{l}
d^{\prime} q_{0} \\
d^{\prime} q_{1} \\
d^{\prime} q_{2} \\
d^{\prime} q_{3}
\end{array}\right]=\frac{1}{2}\left[\begin{array}{cccc}
0 & -\omega_{1}^{\prime} & -\omega_{2}^{\prime} & -\omega_{3}^{\prime} \\
\omega_{1}^{\prime} & 0 & \omega_{3}^{\prime} & -\omega_{2}^{\prime} \\
\omega_{2}^{\prime} & -\omega_{3}^{\prime} & 0 & \omega_{1}^{\prime} \\
\omega_{3}^{\prime} & \omega_{2}^{\prime} & -\omega_{1}^{\prime} & 0
\end{array}\right]\left[\begin{array}{l}
q_{0} \\
q_{1} \\
q_{2} \\
q_{3}
\end{array}\right] .
$$

The model of the three-spherical motion by using quaternions created above is structured according to the informations in [(Blaschke,1960), (Hanson,2006)].

\subsection{Velocities in the Motion:}

Let us assume any quaternionic point, whose coordinates are $x_{0}, x_{1}, x_{2}, x_{3}$ according to the attribution system, is $X$. The $\mathrm{X}$ point denoted by $\mathbf{x}(t)=x_{0} q_{0}+x_{1} q_{1}+x_{2} q_{2}+x_{3} q_{3}$ is a quaternionic point on the three sphere. If the $X$ point is specifically on the unit sphere, then it would have the relation $\|\mathbf{x}\|^{2}=x_{0}^{2}+x_{1}^{2}+x_{2}^{2}+x_{3}^{2}=1$. Unlike the $K$ sphere, we will regard the velocity of the $X$ point as relative velocity and it is obtained by $v_{r}=\frac{d \mathbf{x}}{d t}$. So, the relative velocity vector $K$ sphere is calculated by

$d \mathbf{x}=\frac{1}{2}\left[\begin{array}{l}\left(x_{1} \omega_{1}+x_{2} \omega_{2}+x_{3} \omega_{3}+d x_{0}\right) q_{0}+\left(-x_{0} \omega_{1}-x_{2} \omega_{3}+x_{3} \omega_{2}+d x_{1}\right) q_{1} \\ +\left(-x_{0} \omega_{2}+x_{1} \omega_{3}-x_{3} \omega_{1}+d x_{2}\right) q_{2}+\left(-x_{0} \omega_{3}-x_{1} \omega_{2}+x_{2} \omega_{1}+d x_{3}\right) q_{3}\end{array}\right]$.

If $\mathbf{v}_{\mathbf{r}}=0$ or $d \mathbf{x}=0$, both are the same thing nonetheless; the constancy condition of the $X$ point in the $K$ sphere is represented by the following equations;

$d x_{0}=-x_{1} \omega_{x}-x_{2} \omega_{y}-x_{3} \omega_{z}, \quad d x_{1}=x_{0} \omega_{x}-x_{2} \omega_{z}+x_{3} \omega_{y}$,

$d x_{2}=x_{0} \omega_{y}-x_{1} \omega_{z}+x_{3} \omega_{x}, \quad d x_{3}=x_{0} \omega_{z}+x_{1} \omega_{y}-x_{2} \omega_{x}$.

Similarly, the change in the $X$ point according to the $K^{\prime}$ sphere is

$d^{\prime} \mathbf{x}=\frac{1}{2}\left[\begin{array}{l}\left(x_{1} \omega_{1}^{\prime}+x_{2} \omega_{2}^{\prime}+x_{3} \omega_{3}^{\prime}+d x_{0}\right) q_{0}+\left(-x_{0} \omega_{1}^{\prime}-x_{2} \omega_{3}^{\prime}+x_{3} \omega_{2}^{\prime}+d x_{1}\right) q_{1} \\ +\left(-x_{0} \omega_{2}^{\prime}+x_{1} \omega_{3}^{\prime}-x_{3} \omega_{1}^{\prime}+d x_{2}\right) q_{2}+\left(-x_{0} \omega_{3}^{\prime}-x_{1} \omega_{2}^{\prime}+x_{2} \omega_{1}^{\prime}+d x_{3}\right) q_{3}\end{array}\right]$

What we have calculated here is the "absolute velocity vector", which is the velocity of the $X$ point in the $K^{\prime}$ sphere and is represented by $\mathbf{v}_{\mathbf{a}}=\frac{d^{\prime} \mathbf{x}}{d t}$. Given that $\mathbf{v}_{\mathbf{a}}=0$ or $d^{\prime} \mathbf{x}=0$, the $X$ point in the $K^{\prime}$ sphere is constant. The constancy condition of the $X$ point in the $K^{\prime}$ sphere can be shown by the following equations;

$d x_{0}=-x_{1} \omega_{1}^{\prime}-x_{2} \omega_{2}^{\prime}-x_{3} \omega_{3}^{\prime}, \quad d x_{1}=x_{0} \omega_{1}^{\prime}-x_{2} \omega_{3}^{\prime}+x_{3} \omega_{2}^{\prime}$,

$d x_{2}=x_{0} \omega_{2}^{\prime}-x_{1} \omega_{3}^{\prime}+x_{3} \omega_{1}^{\prime}, \quad d x_{3}=x_{0} \omega_{3}^{\prime}+x_{1} \omega_{2}^{\prime}-x_{2} \omega_{1}^{\prime}$. 
If the $X$ point is constant in the $K$ sphere, its velocity according to $K$ is named as the "dragging velocity" and given that $d_{f} \mathbf{x}=d^{\prime} x-d x$, we will use $\mathbf{v}_{\mathbf{f}}=\frac{d_{f} \mathbf{x}}{d t}$. The dragging velocity vector is calculated by

$d_{f} \mathbf{x}=d^{\prime} \mathbf{x}-d \mathbf{x}=\frac{1}{2}\left[\begin{array}{l}\left(x_{1} \psi_{1}+x_{2} \psi_{2}+x_{3} \psi_{3}\right) q_{0}+\left(-x_{0} \psi_{1}-x_{2} \psi_{3}+x_{3} \psi_{2}\right) q_{1} \\ +\left(-x_{0} \psi_{2}+x_{1} \psi_{3}-x_{3} \psi_{1}\right) q_{2}+\left(-x_{0} \psi_{3}-x_{1} \psi_{2}-x_{2} \psi_{1}\right) q_{3}\end{array}\right]$

where the vector $\psi_{i}=\omega_{i}^{\prime}-\omega_{i}$ is called the Pfaf vector.

Now, if the quaternionic point $X$ is taken with a SLERP in Eq.(3), Eq.(10) and Eq.(11), then the constancy condition of the $X$ point in the $K$ sphere is obtained by

$$
\begin{aligned}
& d x_{0}=-\left(x_{A 1} \omega_{1}+x_{A 2} \omega_{2}+x_{A 3} \omega_{3}\right) \frac{\sin ((1-t) \phi)}{\sin \phi}-\left(x_{B 1} \omega_{1}+x_{B 2} \omega_{2}+x_{B 3} \omega_{3}\right) \frac{\sin (t \phi)}{\sin \phi}, \\
& d x_{1}=\left(x_{A 0} \omega_{1}-x_{A 2} \omega_{2}+x_{A 3} \omega_{3}\right) \frac{\sin ((1-t) \phi)}{\sin \phi}+\left(x_{B 0} \omega_{1}-x_{B 2} \omega_{3}+x_{B 3} \omega_{2}\right) \frac{\sin (t \phi)}{\sin \phi} \\
& d x_{2}=\left(x_{A 0} \omega_{2}-x_{A 1} \omega_{3}+x_{A 3} \omega_{1}\right) \frac{\sin ((1-t) \phi)}{\sin \phi}+\left(x_{B 0} \omega_{2}-x_{B 1} \omega_{3}+x_{B 3} \omega_{1}\right) \frac{\sin (t \phi)}{\sin \phi} \\
& d x_{3}=\left(x_{A 0} \omega_{3}+x_{A 1} \omega_{2}-x_{A 2} \omega_{1}\right) \frac{\sin ((1-t) \phi)}{\sin \phi}+\left(x_{B 0} \omega_{3}+x_{B 1} \omega_{2}-x_{B 2} \omega_{1}\right) \frac{\sin (t \phi)}{\sin \phi}
\end{aligned}
$$

where $q_{A}=x_{A 0} q_{0}+x_{A 1} q_{1}+x_{A 2} q_{2}+x_{A 3} q_{3}$ and $q_{B}=x_{B 0} q_{0}+x_{B 1} q_{1}+x_{B 2} q_{2}+x_{B 3} q_{3}$ are starting and ending quaternionic points on three-sphere. Similarly, the constancy condition of the $X$ point in the $K^{\prime}$ sphere is

$$
\begin{aligned}
& d x_{0}=-\left(x_{A 1} \omega_{1}^{\prime}+x_{A 2} \omega_{2}^{\prime}+x_{A 3} \omega_{3}^{\prime}\right) \frac{\sin ((1-t) \phi)}{\sin \phi}-\left(x_{B 1} \omega_{1}^{\prime}+x_{B 2} \omega_{2}^{\prime}+x_{B 3} \omega_{3}^{\prime}\right) \frac{\sin (t \phi)}{\sin \phi} \\
& d x_{1}=\left(x_{A 0} \omega_{1}^{\prime}-x_{A 2} \omega_{3}^{\prime}+x_{A 3} \omega_{2}^{\prime}\right) \frac{\sin ((1-t) \phi)}{\sin \phi}+\left(x_{B 0} \omega_{1}^{\prime}-x_{B 2} \omega_{3}^{\prime}+x_{B 3} \omega_{2}^{\prime}\right) \frac{\sin (t \phi)}{\sin \phi}, \\
& d x_{2}=\left(x_{A 0} \omega_{2}^{\prime}-x_{A 1} \omega_{3}^{\prime}+x_{A 3} \omega_{1}^{\prime}\right) \frac{\sin ((1-t) \phi)}{\sin \phi}+\left(x_{B 0} \omega_{2}^{\prime}-x_{B 1} \omega_{3}^{\prime}+x_{B 3} \omega_{1}^{\prime}\right) \frac{\sin (t \phi)}{\sin \phi} \\
& d x_{3}=\left(x_{A 0} \omega_{3}^{\prime}+x_{A 1} \omega_{2}^{\prime}-x_{A 2} \omega_{1}^{\prime}\right) \frac{\sin ((1-t) \phi)}{\sin \phi}+\left(x_{B 0} \omega_{3}^{\prime}+x_{B 1} \omega_{2}^{\prime}-x_{B 2} \omega_{1}^{\prime}\right) \frac{\sin (t \phi)}{\sin \phi}
\end{aligned}
$$

If the starting and ending points of the SLERP are sellected with $q_{A}=(1,0,0,0)$ and $q_{B}=\left(\cos \frac{\theta}{2}, \mathbf{n} \sin \frac{\theta}{2}\right)$ where $\cos \phi=\cos \frac{\theta}{2}$, then the point $X$ on three-sphere is formed by $X(t)=\left(\cos \left(\frac{t \theta}{2}\right), \mathbf{n} \sin \left(\frac{t \theta}{2}\right)\right)$ from Eq.(4). Hence using the Eq. (10), the relative velocity vector is found by

$$
d \mathbf{x}=\left[\begin{array}{l}
q_{0}\left(n_{1} \sin \frac{t \theta}{2} \omega_{1}+n_{2} \sin \frac{t \theta}{2} \omega_{2}+n_{3} \sin \frac{t \theta}{2} \omega_{3}+d x_{0}\right)+q_{1}\left(-\cos \frac{t \theta}{2} \omega_{1}-n_{2} \sin \frac{t \theta}{2} \omega_{3}+n_{3} \sin \frac{t \theta}{2} \omega_{2}+d x_{1}\right) \\
+q_{2}\left(-\cos \frac{t \theta}{2} \omega_{2}+n_{1} \sin \frac{t \theta}{2} \omega_{3}-n_{3} \sin \frac{t \theta}{2} \omega_{1}+d x_{2}\right)+q_{3}\left(-\cos \frac{t \theta}{2} \omega_{3}-n_{1} \sin \frac{t \theta}{2} \omega_{2}+n_{2} \sin \frac{t \theta}{2} \omega_{1}+d x_{3}\right)
\end{array}\right],
$$

and from Eq.(11), the constancy condition of the $X$ point in the $K$ sphere is obtained by

$$
\begin{aligned}
& d x_{0}=-\sin \frac{t \theta}{2}\left(n_{1} \omega_{1}+n_{2} \omega_{2}+n_{3} \omega_{3}\right), \quad d x_{1}=\cos \frac{t \theta}{2} \omega_{1}+\sin \frac{t \theta}{2}\left(n_{2} \omega_{3}-n_{3} \omega_{2}\right), \\
& d x_{2}=\cos \frac{t \theta}{2} \omega_{2}+\sin \frac{t \theta}{2}\left(n_{3} \omega_{1}-n_{1} \omega_{3}\right), \quad d x_{3}=\cos \frac{t \theta}{2} \omega_{3}+\sin \frac{t \theta}{2}\left(n_{1} \omega_{2}-n_{2} \omega_{1}\right),
\end{aligned}
$$


Furthermore, from Eq.(12) the relative velocity vector in $K^{\prime}$ sphere is

$$
d^{\prime} \mathbf{x}=\left[\begin{array}{l}
q_{0}\left(n_{1} \sin \frac{t \theta}{2} \omega_{1}^{\prime}+n_{2} \sin \frac{t \theta}{2} \omega_{2}^{\prime}+n_{3} \sin \frac{t \theta}{2} \omega_{3}^{\prime}+d x_{0}\right)+q_{1}\left(-\cos \frac{t \theta}{2} \omega_{1}^{\prime}-n_{2} \sin \frac{t \theta}{2} \omega_{3}^{\prime}+n_{3} \sin \frac{t \theta}{2} \omega_{2}^{\prime}+d x_{1}\right) \\
+q_{2}\left(-\cos \frac{t \theta}{2} \omega_{2}^{\prime}+n_{1} \sin \frac{t \theta}{2} \omega_{3}^{\prime}-n_{3} \sin \frac{t \theta}{2} \omega_{1}^{\prime}+d x_{2}\right)+q_{3}\left(-\cos \frac{t \theta}{2} \omega_{3}^{\prime}-n_{1} \sin \frac{t \theta}{2} \omega_{2}^{\prime}+n_{2} \sin \frac{t \theta}{2} \omega_{1}^{\prime}+d x_{3}\right)
\end{array}\right]
$$

Thus, using Eq.(13) the constancy condition of the $X$ point in the $K^{\prime}$ sphere is calculated by

$$
\begin{aligned}
& d x_{0}=-\sin \frac{t \theta}{2}\left(n_{1} \omega_{1}^{\prime}+n_{2} \omega_{2}^{\prime}+n_{3} \omega_{3}^{\prime}\right), \quad d x_{1}=\cos \frac{t \theta}{2} \omega_{1}^{\prime}+\sin \frac{t \theta}{2}\left(n_{2} \omega_{3}^{\prime}-n_{3} \omega_{2}^{\prime}\right), \\
& d x_{2}=\cos \frac{t \theta}{2} \omega_{2}^{\prime}+\sin \frac{t \theta}{2}\left(n_{3} \omega_{1}^{\prime}-n_{1} \omega_{3}^{\prime}\right), \quad d x_{3}=\cos \frac{t \theta}{2} \omega_{3}^{\prime}+\sin \frac{t \theta}{2}\left(n_{1} \omega_{2}^{\prime}-n_{2} \omega_{1}^{\prime}\right) .
\end{aligned}
$$

Similarly, the dragging velocity vectors should be obtained by the Eq.(14). In a one-parameter $D_{I}$ rotation motion, at the moment of $t$; an infinite-small rotating motion occurs for each $X$ point of the moving system. The Pfaf vector $\boldsymbol{\psi}$ in this rotating motion plays the role of the darboux rotation vector. If $\mathbf{p}^{2}=1$, then $\boldsymbol{\psi}=\mathbf{p} \cdot \sqrt{\psi_{1}^{2}+\psi_{2}^{2}+\psi_{3}^{2}}$ and $\psi= \pm|\psi|=\sqrt{\psi_{1}^{2}+\psi_{2}^{2}+\psi_{3}^{2}}$. The $P$ point shown on the unit sphere via $O P=\mathbf{p}$ is the instantenous rotation pole. So, $p$ as the instantenous rotation pole is characterized with the drift velocity being zero. Both the $P$ rotation pole and its $\hat{P}$ counterpoint are constant at the moment of $t \in\left(t_{0}, t_{1}\right)$. So, the quaternion interpolation of the pole curves and its representation during the spherical motion can be thought to be formulated via the following big circles of the sphere that intersects with the $P$ and $\hat{P}$ points

$$
\operatorname{SLERP}(\mathbf{p}, \hat{\mathbf{p}}, t)=\mathbf{p} \sin \frac{(1-t) \phi}{\sin \phi}+\hat{\mathbf{p}} \frac{\sin t \phi}{\sin \phi} .
$$

\subsection{For The Canonical Attribution System:}

Let us assume as a specific choice that $(0, \mathbf{p})=\left(0, \mathbf{q}_{\mathbf{3}}\right)$ and $\mathbf{p}$ is perpendicular to $\mathbf{q}_{\mathbf{1}}$ and $\mathbf{q}_{\mathbf{2}}$ during the spherical motion. Then the following conditions must be enabled;

$\boldsymbol{\psi} . \mathbf{q}_{1}=\psi_{1}=0, \quad \boldsymbol{\psi} . \mathbf{q}_{2}=\psi_{2}=0$.

$\omega_{1}=\omega_{1}^{\prime}, \omega_{2}=\omega_{2}^{\prime}$ as owing to $\psi_{i}=\omega_{i}^{\prime}-\omega_{i}, i=1,2,3$. With this specific choice, the infinite-small rotation angle of the instantenous rotation is simplified as $\psi=\psi_{3}$. So, the instantenous rotation vector is $\boldsymbol{\psi}=\mathbf{q}_{3} \cdot \psi_{3}=\mathbf{q}_{3}\left(\omega_{z}^{\prime}-\omega_{z}\right)$ under the condition that $\psi_{3} \neq 0$. To identify the attribution system with a different method, we will spin the $\mathbf{q}_{1}$ and $\mathbf{q}_{2}$ elements of the quaternionic frame around $\mathbf{q}_{3}$ inasmuch as $\varphi$. By benefitting from the easy use of quaternions, we will show this rotation process with the quaternion $q=\cos \frac{\varphi}{2}+\mathbf{q}_{3} \sin \frac{\varphi}{2}$. Then, we can represent the elements of the quaternionic frame using the following relations;

$$
\begin{aligned}
& q_{0}^{*}=\left(0, \mathbf{q}_{\mathbf{0}}^{*}\right)=q \cdot\left(0, \mathbf{q}_{\mathbf{0}}\right) \cdot q^{-1}, q_{1}^{*}=\left(0, \mathbf{q}_{\mathbf{1}}^{*}\right)=q \cdot\left(0, \mathbf{q}_{\mathbf{1}}\right) \cdot q^{-1} \\
& q_{2}^{*}=\left(0, \mathbf{q}_{\mathbf{2}}^{*}\right)=q \cdot\left(0, \mathbf{q}_{\mathbf{2}}\right) \cdot q^{-1}, q_{3}^{*}=\left(0, \mathbf{q}_{3}^{*}\right)=q \cdot\left(0, \mathbf{q}_{\mathbf{3}}\right) \cdot q^{-1}
\end{aligned}
$$

Here, the rotated quaternion frame is represented via $\left\{q_{0}^{*}=1, q_{1}^{*}, q_{2}^{*}, q_{3}^{*}\right\}$. As a result, when $\omega_{1}=0$ in the matrix equations (8) and (9), the system of the differential equations belonging to the attribution system are represented according to $K$ with the following equation; 
$\left[\begin{array}{l}d q_{0} \\ d q_{1} \\ d q_{2} \\ d q_{3}\end{array}\right]=\frac{1}{2}\left[\begin{array}{cccc}0 & 0 & -\omega_{2} & -\omega_{3} \\ 0 & 0 & \omega_{3} & -\omega_{2} \\ \omega_{2} & -\omega_{3} & 0 & 0 \\ \omega_{3} & \omega_{2} & 0 & 0\end{array}\right]\left[\begin{array}{l}q_{0} \\ q_{1} \\ q_{2} \\ q_{3}\end{array}\right]$,

and according to $K^{\prime}$, with the following equation;

$\left[\begin{array}{l}d^{\prime} q_{0} \\ d^{\prime} q_{1} \\ d^{\prime} q_{2} \\ d^{\prime} q_{3}\end{array}\right]=\frac{1}{2}\left[\begin{array}{cccc}0 & 0 & -\omega_{2}^{\prime} & -\omega_{3}^{\prime} \\ 0 & 0 & \omega_{3}^{\prime} & -\omega_{2}^{\prime} \\ \omega_{2}^{\prime} & -\omega_{3}^{\prime} & 0 & 0 \\ \omega_{3}^{\prime} & \omega_{2}^{\prime} & 0 & 0\end{array}\right]\left[\begin{array}{l}q_{0} \\ q_{1} \\ q_{2} \\ q_{3}\end{array}\right]$.

A $(P)$ curve that is known as a moving pole curve is drawn with the one-parameter $D_{I H}$ motion on the moving $K$ sphere in the canonical attribution system. The $P$ rotation pole is in motion and its possessed velocity vectors are the same at every moment when the $(P)$ and $\left(P^{\prime}\right)$ pole curves are drawn in a respective manner. The spherical $(P)$ moving pole curve of $K$ rolls without slipping on the $\left(P^{\prime}\right)$ constant pole curve of $K^{\prime}$ during a one-parameter spherical $D_{I}$ motion.

\subsection{A Numeric Example}

First, we obtain the basis system of two intertwined sphere motions, after calculating the spherical bases of the $K$ and $K^{\prime}$ spheres that were established with the help of quaternion rotations of the $\mathrm{x}, \mathrm{y}, \mathrm{z}$ coordinate axes that we already know. To rotate the $\mathrm{x}, \mathrm{y}, \mathrm{z}$ axes for the $K$ sphere around the axis $\tilde{\mathbf{n}}=(1,0,0)$ inasmuch as the angle $\frac{\pi}{6}$, the angle $\tilde{\theta}=\frac{\pi}{3}$ is used; thereby we find the quaternion that will form the $\left\{O, e_{0}, e_{1}, e_{2}, e_{3}\right\}$ basis system as;

$$
\begin{aligned}
\tilde{q} & =\cos \frac{\tilde{\theta}}{2}+\tilde{\mathbf{n}} \sin \frac{\tilde{\theta}}{2}=\cos \frac{\pi}{6}+(1,0,0) \sin \frac{\pi}{6} \\
& =\frac{\sqrt{3}}{2}+(1,0,0) \cdot \frac{1}{2}=\left(\frac{\sqrt{3}}{2}, \frac{1}{2}, 0,0\right)
\end{aligned}
$$

If so, then by calculating the quaternion multiplications of the $\left\{e_{0}, e_{1}, e_{2}, e_{3}\right\}$ bases with the help of the Mathematica program's "Quaternion package", we find the bases as;

$e_{0}=\tilde{q}(1, \mathbf{0}) \tilde{q}^{-1}=\left(\frac{\sqrt{3}}{2}, \frac{1}{2}, 0,0\right)(1,0,0,0)\left(\frac{\sqrt{3}}{2},-\frac{1}{2}, 0,0\right)=(1,0,0,0)$

$e_{1}=\tilde{q}(1, \mathbf{x}) \tilde{q}^{-1}=\left(\frac{\sqrt{3}}{2}, \frac{1}{2}, 0,0\right)(0,1,0,0)\left(\frac{\sqrt{3}}{2},-\frac{1}{2}, 0,0\right)=(0,1,0,0)$

$e_{2}=\tilde{q}(1, \mathbf{y}) \tilde{q}^{-1}=\left(\frac{\sqrt{3}}{2}, \frac{1}{2}, 0,0\right)(0,0,1,0)\left(\frac{\sqrt{3}}{2},-\frac{1}{2}, 0,0\right)=\left(0,0, \frac{1}{2}, \frac{\sqrt{3}}{2}\right)$

$e_{3}=\tilde{q}(1, \mathbf{z}) \tilde{q}^{-1}=\left(\frac{\sqrt{3}}{2}, \frac{1}{2}, 0,0\right)(0,0,0,1)\left(\frac{\sqrt{3}}{2},-\frac{1}{2}, 0,0\right)=\left(0,0,-\frac{\sqrt{3}}{2}, \frac{1}{2}\right)$

Here, we can see that the quaternionic bases provide the equations $e_{1}^{2}=e_{2}^{2}=e_{3}^{2}=-1 ; e_{1} e_{2}=e_{3}, e_{3} e_{2}=e_{1}, e_{3} e_{1}=e_{2}$. Now, off to calculate the second $\left\{O, e_{0}^{\prime}, e_{1}^{\prime}, e_{2}^{\prime}, e_{3}^{\prime}\right\}$ quaternionic frame; to rotate the $\mathrm{x}, \mathrm{y}, \mathrm{z}$ axes for the $K^{\prime}$ sphere around the axis $\hat{\mathbf{n}}=(1,0,0)$ inasmuch as the angle $\pi / 2$, the angle $\hat{\theta}=\pi$ is used; thereby we find the quaternion that will form the $\left\{O, e_{0}^{\prime}, e_{1}^{\prime}, e_{2}^{\prime}, e_{3}^{\prime}\right\}$ quaternionic basis system as; 


$$
\begin{aligned}
\hat{q} & =\cos \frac{\hat{\theta}}{2}+\hat{\mathbf{n}} \sin \frac{\hat{\theta}}{2}=\cos \frac{\pi}{2}+(1,0,0) \sin \frac{\pi}{2} \\
& =0+(1,0,0) \cdot 1=(0,1,0,0)
\end{aligned}
$$

Then, by calculating the quaternion multiplications of the $\left\{e_{0}, e_{1}, e_{2}, e_{3}\right\}$ bases with the help of the Mathematica program's "Quaternion package", we find said bases as;

$e_{0}^{\prime}=\hat{q}(1, \mathbf{0}) \hat{q}^{-1}=(0,1,0,0)(1,0,0,0)(0,-1,0,0)=(1,0,0,0)$

$e_{1}^{\prime}=\hat{q}(1, \mathbf{x}) \hat{q}^{-1}=(0,1,0,0)(0,1,0,0)(0,-1,0,0)=(0,1,0,0)$

$e_{2}^{\prime}=\hat{q}(1, \mathbf{y}) \hat{q}^{-1}=(0,1,0,0)(0,0,1,0)(0,-1,0,0)=(0,0,-1,0)$

$e_{3}^{\prime}=\hat{q}(1, \mathbf{z}) \hat{q}^{-1}=(0,1,0,0)(0,0,0,1)(0,-1,0,0)=(0,0,0,-1)$

Here, we can see that the quaternionic bases provide the conditions $e_{1}^{\prime 2}=e_{2}^{\prime 2}=e_{3}^{\prime 2}=-1$ and $e_{1}^{\prime} e_{2}^{\prime}=e_{3}^{\prime}, e_{3}^{\prime} e_{2}^{\prime}=e_{1}^{\prime}$, $e_{3}^{\prime} e_{1}^{\prime}=e_{2}^{\prime}$. Now, we will form the third $\left\{O, q_{0}, q_{1}, q_{2}, q_{3}\right\}$ quaternionic root, in order to examine the spherical motion. To rotate the $\mathrm{x}, \mathrm{y}, \mathrm{z}$ axes around the axis $\widehat{\mathbf{n}}=(1,0,0)$ inasmuch as the angle 0 , the angle $\widehat{\theta}=0$ is used; thereby we find the quaternion

$$
\begin{aligned}
\hat{q} & =\cos \frac{\hat{\theta}}{2}+\hat{\mathbf{n}} \sin \frac{\hat{\theta}}{2}=\cos 0+(1,0,0) \sin 0 \\
& =1+(1,0,0) \cdot 0=(1,0,0,0)
\end{aligned}
$$

that will form the $\left\{O, q_{0}, q_{1}, q_{2}, q_{3}\right\}$ quaternionic basis system as;

$$
\begin{aligned}
& q_{0}=\widehat{q}(1, \mathbf{0}) \hat{q}^{-1}=(1,0,0,0)(1,0,0,0)(1,0,0,0)=(1,0,0,0) \\
& q_{1}=\widehat{q}(1, \mathbf{x}) \hat{q}^{-1}=(1,0,0,0)(0,1,0,0)(1,0,0,0)=(0,1,0,0) \\
& q_{2}=\widehat{q}(1, \mathbf{y}) \widehat{q}^{-1}=(1,0,0,0)(0,0,1,0)(1,0,0,0)=(0,0,1,0) \\
& q_{3}=\hat{q}(1, \mathbf{z}) \widehat{q}^{-1}=(1,0,0,0)(0,0,0,1)(1,0,0,0)=(0,0,0,1)
\end{aligned}
$$

Here, we can see that the conditions $q_{0}=1, q_{1}^{2}=q_{2}^{2}=q_{3}^{2}=-1, q_{1} q_{2}=q_{3}, q_{3} q_{2}=q_{1}, q_{3} q_{1}=q_{2}$ are enabled using the properties of quaternion. Then, the matrices of the tribasic system for the sphere motion are as follows;

$\left[e_{0}, e_{1}, e_{2}, e_{3}\right]=\left[\begin{array}{cccc}1 & 0 & 0 & 0 \\ 0 & 1 & 0 & 0 \\ 0 & 0 & 1 / 2 & -\sqrt{3} / 2 \\ 0 & 0 & \sqrt{3} / 2 & 1 / 2\end{array}\right],\left[e_{0}^{\prime}, e_{1}^{\prime}, e_{2}^{\prime}, e_{3}^{\prime}\right]=\left[\begin{array}{cccc}1 & 0 & 0 & 0 \\ 0 & 1 & 0 & 0 \\ 0 & 0 & -1 & 0 \\ 0 & 0 & 0 & -1\end{array}\right]\left[q_{0}, q_{1}, q_{2}, q_{3}\right]=\left[\begin{array}{cccc}1 & 0 & 0 & 0 \\ 0 & 1 & 0 & 0 \\ 0 & 0 & 1 & 0 \\ 0 & 0 & 0 & 1\end{array}\right]$

Now, let us specifically choose an $X$ point on the unit sphere;

$$
X(t)=\operatorname{SLERP}\left(q_{A}, q_{B}, t\right)=\left(\cos \frac{t \theta}{2}+\mathbf{n} \sin \frac{t \theta}{2}\right) .
$$

Here, if the direction vector and angle are chosen as $\mathbf{n}=(1,0,0)$ and $\theta=\frac{\pi}{3}$, then $X(t)=\left(\cos \frac{t \pi}{6}+\mathbf{n} \sin \frac{t \pi}{6}\right) \cdot$ The angular velocity vector of the spherical motion is found by

$\boldsymbol{\omega}=2 .\left(q_{0} d q-q d q_{0}-q \times d q\right)=\left(\frac{\pi}{3}, 0,0\right)$. Then, the relative velocity vector of the motion at each $\mathrm{t}$ moment is $d \mathbf{x}=\left[q_{0}\left(\frac{\pi}{3} \sin \frac{\pi t}{6}+d x_{0}\right)+q_{1}\left(-\cos \frac{\pi t}{6}+d x_{1}\right)+q_{2}\left(d x_{2}\right)+q_{3}\left(d x_{3}\right)\right]$. 
Constancy conditions of the $X$ point chosen for $\mathbf{v}_{r}=\mathbf{0}$ are

$d x_{0}=-\frac{\pi}{3} \sin \frac{t \pi}{6}, d x_{1}=\cos \frac{t \pi}{6}, d x_{2}=0, d x_{3}=0$.

Other velocity vectors formed during spherical motion can be calculated in a similar manner.

\section{REFERENCES}

Hamilton, W. (1853). Lectures on Quaternions. Hodges Smith\&Co.Dublin 350pp.

Garnier, R. (1956). Cours de Cinematique, Tome II: Roulement et Vibration-La Formule de Savary et son Extension a l'Espace. Gauthier-Villars, Paris.

Müller, HR. (1963). Kinematik Dersleri, Ankara University.

Blaschke, W. (1960). Kinematics and Quaternions. VEB Verlag, Berling Math. Monographien Bd.4.

Bottema, O. \& Roth, B. (1978). Theoretical Kinematics, Amsterdam: North-Holland Publishers Co.

Hacısalihoğlu, H.H.,(1983). Hareket Geometrisi ve Kuaterniyonlar Teorisi, Gazi Universitesi Yayınları, Ankara.

Hanson, A.J. (2006).Visualizing Quaternion, Morgan-Kaufmann, Elsevier, London.

Nixravesh, P.E., R.A. Wehage \& O.K. Kwan, (1985). Euler Parameters in Computational Kinematics and Dynamics, Part, ASME J. Mech. Trans, Aut. Des (107) 358-365.

Larochelle, P. (2000). Approximate Motion Synthesis via Parametric Constraint Manifold Fitting, Advances in Robot Kinematics, Kluwer Acad. Publ., Dordrecht.

Liu X.J., Wang J. \& Gao, F. (2003). Workspace Atlases fort he Design of Spherical 3-DOF Serial Wrist, J. Of Intelligent and Robotic Systems 389-405.

Yang, A.T. \& Freudenstein, F. (1964). Application of dual number quaternion algebra to the analysis of spatrial mechanism, Transactions of the ASNE, 300-308.

Alizade R., Gezgin E.\& Kilit Ö. (2005). A New Method in Computational Kinematics of a Spherical Wrist Motion Through Quaternions, Intl. Workshop on Comp. Kinematics, Cassino, p.32.

Kuşak, H., Çalışkan A., (2011). About Dual Spherical Wrist Motion and its Trajectory Surface as a Ruled Surface, Mathematical and Computational Applications, Mathematical and Computational Applications, Vol. 16, No. 1, pp. 309-316.

Shoemake K., (1985). Animating Rotation with Quaternion Curves, in Computer Graphics, SIGGRAPH'85 Proceedings, Vol.19, pp 245-254.

Shoemake K., (1987). Quaternion Calculus for Animation, Siggraph’ 89 Course 23:Math for SIGGRAPH.

Hanson A.J. (2006). Visualizing Quaternions, Morgan-Kaufmann, ELsevier, London. 